\title{
TBILISI RADIOCARBON DATES III
}

\section{A A BURCHULADZE, L D GEDEVANISHVILI, and G I TOGONIDZE}

\author{
Radiocarbon Laboratory, Tbilisi State University, Tbilisi, USSR
}

The Radiocarbon Laboratory under the Chair of Nuclear physics of Tbilisi State University is engaged in studies of radiocarbon variation in the atmosphere and mineral waters and determination of the absolute age of archaeologic, geologic, botanical, and other samples. This list reports dates of archaeologic and geologic specimens only. Gas counting and liquid scintillation methods are used for dating.

Initially $\mathrm{CO}_{2}$ activity was determined by proportional counting. Results of the first measurement were published earlier (Burchuladze, 1968). At present, the absolute age of most samples is determined in laboratory by the liquid scintillation method. For this, a 3-channel scintillation spectrometer SL-30 of "Intertechnique" was used, and benzene $\mathrm{C}_{6} \mathrm{H}_{6}$ served as scintillation solvent. Benzene is synthesized according to the modified method of Barker (1969). The pretreated sample is burnt out instantaneously in the calorimetric "bomb" under high pressure of oxygen, and the obtained $\mathrm{CO}_{2}$ needs no further purification. Acetylene is obtained from $\mathrm{CO}_{2}$ through lithium carbide by means of dissociation with artesian water (from Tbilisi) and then is transformed into benzene $\left(\mathrm{C}_{6} \mathrm{H}_{6}\right)$ with the help of alumosilicate catalyst, activated by vanadium pentoxide with a high yield of benzene $(\sim 90 \%)$. Usually $5 \mathrm{ml}$ of benzene synthesized from the sample in the vessel with minimum content of potassium is dissolved in $5 \mathrm{ml}$ of "dead" benzene with the content of $4 \mathrm{~g} / 1$ PPO and $.1 \mathrm{~g} / 1$ POPOP. For one of the ${ }^{14} \mathrm{C}$ channels an SL-30 spectrometer is used for measurements in which the interval with high quality and effectiveness of $64.9 \%$ is selected.

Volume of scintillation solvent of benzene is $10 \mathrm{ml}$, the count of "dead carbon" (background) is $11.3 \mathrm{cpm}$, the count of modern carbon $76.6 \mathrm{cpm}$ (the reference sample of the modern carbon was prepared in the laboratory of the Inst Geol Acad Sci USSR). Modern carbon is controlled by NBS oxalic acid (USA).

Measuring time of sample activity was 30 to 50 hours. Obtained data are registered by digital printers each $100 \mathrm{~min}$. After 5 to 6 samples are measured, the activity of modern and dead carbon is checked. Statistical accuracy of measurements for various samples is $2 \sigma$ to $3 \sigma$. Calculations are based on the ${ }^{14} \mathrm{C}$ half-life, $5568 \pm 30$ years, with values relative to 1950 . Below are given the dating results made by gas and scintillation methods, and the characteristics of those samples as well.

\section{ARCHAEOLOGIC SAMPLES}

\section{TB-7. Mestia, Georgian SSR}

$900 \pm 110$ AD 1050

Wooden beams and boards. Wall of Svani tower, ancient tower of Koldans, Muzhali village, Mestia region. Archaeol date: Middle age. 
Subm by G Chitaya, Inst History, Archaeol \& Ethnog, Georgian Acad Sci.

\section{TB-84. Mestia, Georgian SSR}

Cereals, Kvanchiantkari village, Mestia region. Subm by I Tumajanov and L Gogichaishvili, Inst Botanics, Acad Sci, GSSR.

\section{TB-10. Novgorod region, RSFSR}

Wood (birch) remains of bark. Lake peat, $2.3 \mathrm{~m}$ deep, Novgorod region. Dated to compare with results from radiocarbon lab of Geol Chem: Mo-346 $(5860 \pm 210)$. Subm by A Devirtz, Inst Geochem \& Analytical Chem, Vernadsky Acad Sci USSR.

\section{TB-11. Tsulukidze, Georgian SSR}

Wood with bull bones incorporated into it. Mus Regional Studies, City of Tsulukidze. Subm by P Pirpilashvili, State Mus Georgia.

\section{TB-24. Uplistsikhe, Georgian SSR}

$1925 \pm 175$

Semi-carbonized tree. Field no. AI, Uplistsikhe village, Gori region. Archaeol date: Late Bronze age. Subm by D Khakhutaishvili, Inst History, Archaeol \& Ethnog, Acad Sci GSSR.

\section{TB-25. Uplistsikhe, Georgian SSR}

$$
1130 \pm 125
$$

Semi-carbonized tree. Field no. A2. From same location (TB-24). Subm by D Khakhutaishvili.

\section{TB-26. "Sabid-Akhcha", Georgian SSR}

Wood from wooden 4-wheeled chariot, Im deep. Bartow "Sabid-Akhcha" Tsalka Plateau. Archaeol da: Trep. Barrow no. 5, by O Japaridze, Tbilisi State Univ.

\section{TB-27. "Imiris Gora", Georgian SSR}

$6300 \pm 120$

Wood remains of carbonized wooden structure of building span, "Imiris-Gora", Shulaveri village, Marneuli region. Archaeol date: Chalcolithic epoch. Subm by O Japaridze and A Javakhishvili, State Mus Georgia.

\section{TB-29. "Khizanaant-Gora", Georgian SSR}

Cereals, "Khizanaant-Gora”, Urbnisi village, Kareli region. Archaeol date: Early Bronze age. Subm by Y Kikvidze, State Mus Georgia.

\section{TB-30. Tetri-Tskaro, Georgian SSR}

$3330 \pm 60$

Wood, remains of tomb, $10 \mathrm{~m}$ deep, Bedeni upland, Tetri-Tskaro. 
Archaeol date: Bronze age. Subm by A Apakidze and G Gobejishvili, Inst History, Archaeol \& Ethnog, Acad Sci GSSR.

TB-31. Metekhi, Georgian SSR

$3235 \pm 60$

1285 BC

Wood, 5m deep, Burial no. 6, Metekhi village, Kaspi region. Archaeol date: Middle Bronze age. Subm by $\mathrm{Z}$ Shatberashvili, Inst History, Archaeol \& Ethnog, Acad Sci GSSR.

\section{TB-32. Mtskheta, Georgiian SSR}

$1670 \pm 50$

Coal, 5m deep, City gates, Mtskheta. Archaeol date: Early Middle ages. Subm by A Kalandadze, Inst History, Archaeol \& Ethnog, Acad Sci GSSR.

TB-34. "Treligora", Georgian SSR

$2565 \pm 60$

$615 \mathrm{BC}$

Coal, 1.2 to $1.5 \mathrm{~m}$ deep, Burial no. 9, "Treligora", Digomi, Tbilisi. Archaeol date: Iron age. Subm by R Abramishvili, Inst History, Archaeol \& Ethnog, Acad Sci GSSR.

\section{TB-37. "Treligora", Georgian SSR}

$2730 \pm 130$

Coal, $4 \mathrm{~m}$ deep. From same location as TB-34, from stone burial, no. 16. Archaeol date: Iron age. Subm by R Abramishvili.

\section{TB-35. "Darbazi", Georgian SSR}

Wood from peasant's house "Darbazi”, Chachkari village, Aspindza region. Archaeol date: Middle ages. Subm by G Chtaya, Inst History, Archaeol \& Ethnog, Acad Sci GSSR.

\section{TB-36. "Samnateo", Georgian SSR}

$740 \pm 50$

Wooden piece of beam, "Samnateo", Sanctuary Kotia, Tsitelauri village, Pshavi, Dusheti region. Archaeol date: Middle ages. Subm by G Chitaya, Inst History, Archaeol \& Ethnog, Acad Sci GSSR.

\section{TB-38. The mummy, Egypt}

$4330 \pm 160$

2380 BC

Bandages of Egyptian mummy, Tarkhan II, Cairo, Egypt. Sample was subm by Univ Birmingham lab for control dating; it was previously dated by other labs: Arizona: A-569 (4295 \pm 90$)$, Univ California: UCLA739 (4265 \pm 80$)$, British Mus: BM-203 (4150 \pm 110$)$, Univ Birmingham: Birm-20 (4224 \pm 97$)$, Natl Physical Lab: NPL (4310 \pm 90$)$.

\section{TB-40. Akhaltsikhe, Georgian SSR}

$$
990 \pm 90
$$

\section{AD 960}

Remains of cloth from mummified corpse from one of bricked-up caves, Janisi village, Akhaltsikhe region. Subm by P Pirpilashvili, Research Inst Traumatology \& Orthopedics, Ministry Public Health, Georgian SSR. 
TB-41. Tsutskhvati, Georgian SSR

Bones (cave-bear) from Bison cave, Tsutskhvati village, Tkibuli region. Gave remains are of upper Pleistocene. Subm by L Maruashvili, Inst Geog, Acad Sci GSSR.

\section{TB-49. "Choga", Georgian SSR}

Coal, .35 to .5m deep, "Choga II", First Choga village, Chkhorotski region. Subm by D Khakhutaishvili.

\section{TB-52. "Simagre", Georgian SSR}

$$
1930 \pm 50
$$

Wood from ruins of ancient building, Hill "Simagre", Sakorkio village, Khobi region. Archaeol date: Roman epoch. Subm by G Mikeladze, T Baramidze, Inst History, Archaeol \& Ethnog, Acad Sci, GSSR.

TB-58. "Simagre", Georgian SSR

$980 \pm 40$

Wood, $4 \mathrm{~m}$ deep, from same location as TB-52. From deposits of 1 st terrace over flood land, bank of Rioni R. Subm by T Mikeladze, D Tabidze, Inst History, Archaeol \& Ethnog, Acad Sci, GSSR.

\section{Kulevi series}

TB-60. Kulevi, Georgian SSR

$$
2010 \pm 45
$$

$60 \mathrm{BC}$

Wood, $1.3 \mathrm{~m}$ deep, ancient Kolkhi settlement, 4th cultural layer. Right bank of Khobi R, Kulevi village, Khobi region. Subm by D Khakhutaishvili.

TB-61. Kulevi, Georgian SSR

$$
2260 \pm 45
$$

Wood, $1.5 \mathrm{~m}$ deep, from same location as TB-60. Subm by D Khakhutaishvili.

\section{TB-62. Kulevi, Georgian SSR}

$2150 \pm 45$

Wood, $1.6 \mathrm{~m}$ deep, from same location as TB-60, 61. Subm by D Khakhutaishvili.

\section{TB-68. Tsutskhvati, Georgian SSR}

$605 \pm 40$

\section{AD 1345}

Wood, from Tsutskhvati cave, Tsutskhvati village, Tkibuli region. Subm by L Maruashvili.

\section{TB-72. "Guard Hill", Georgian SSR}




\section{TB-73. "Tetri-Mgvime", Georgian SSR \\ $\mathbf{3 0 2 5} \pm \mathbf{5 0}$ \\ $1075 \mathrm{BC}$}

Coal, $10 \mathrm{~m}$ deep relative to present earth surface in cave, "TetriMgvime”, Legvtadzeebi Hill, Khomuri village, Tskkaltubo region. Archaeol date: Bronze age. Subm by K Kalandadze, Inst History, Archaeol \& Ethnog, Acad Sci, GSSR.

TB-74. "Tsikhi-Gora", Georgian SSR

$1885 \pm 40$

AD 65

Cereals, 1.5m deep. "Tsikhi-Gora", Kavtiskhevi village, Kaspi region. Subm by G Tskitishvili, Inst History, Archaeol \& Ethnog, Acad Sci GSSR.

TB-75. "Tsikhi-Gora", Georgian SSR

$1995 \pm 40$

$45 \mathrm{BC}$

Wood, 3.5m deep, "Tsikhi-Gora", from same location as TB-74. Subm by G Tskitishvili.

TB-80. "Dikha-Gubura", Georgian SSR

$3235 \pm 50$

1285 BC

Wood, 5m deep, "Dikha-Gubura II", left bank of Inguri R, Anaklia village, Zugdidi region. Subm by D Muskhelishvili, Inst History, Archaeol \& Ethnog, Acad Sci, GSSR.

Namcheduri series

TB-81. "Namcheduri", Georgian SSR

$3115 \pm 50$

$1165 \mathrm{BC}$

Wood, $4.4 \mathrm{~m}$ deep, 7th layer, "Namcheduri", right bank of Ochkhamuri R, city of Kobuleti, Ajarian ASSR. Subm by D Khakhutaishvili.

TB-50. "Namcheduri", Georgian SSR

$2770 \pm 45$

820 BC

Wood, 3m deep, "Namcheduri", 5th cultural layer, ancient Kolkhi settlement, right bank Ochkhamuri R, Kobuleti region, Ajarian ASSR. Subm by D Khakhutaishvili.

\section{TB-63. "Namcheduri", Georgian SSR}

$2795 \pm 50$

Wood, $3 \mathrm{~m}$ deep, from same location as TB-50, 4th cultural layer. Subm by D Khakhutaishvili.

\section{TB-82. "Ispani", Georgian SSR}

$4405 \pm 50$

2455 BC

Wood, 2.8 to $3 \mathrm{~m}$ deep in peaty layer, "Ispani", left bank of Shavcheli $\mathrm{R}$, Kobuleti region, Ajarian ASSR. Subm by D Khakhutaishvili.

\section{GEOLOGIC SAMPLES}

\section{TB-33. Tokhliauri, Georgian SSR}

Lake deposits with organic particles, $4.25 \mathrm{~m}$ deep, 2nd terrace of Iozi $\mathrm{R}$, Sagarejo region. Subm by I Tumajanov and L Gogichaishvili. 
Kolkhidka series

TB-42. Kolkhidka, Georgian SSR

$4460 \pm 150$

Peat, $1.8 \mathrm{~m}$ deep in terrace surface, from deposits of New Black Sea terrace, mouth of Kolkhidka R, Gagra, Abkhazian ASSR. Subm by C Janelidze, Inst Geog, Acad Sci, GSSR.

TB-43. Kolkhidka, Georgian SSR

$4280 \pm 60$

Submerged peat, 4 to $5 \mathrm{~m}$ below water surface, from same location as TB-42, from surface of submerged peat horizon. Subm by C Janelidze.

\section{TB-47. Kolkhidka, Georgian SSR}

$7060 \pm 100$

$\mathbf{5 1 1 0 ~ B C}$

Submerged peat, 4 to $5 \mathrm{~m}$ deep, from same location as TB-43. Sample was taken from sea bottom, from lower layers of submerged peaty horizon. Subm by $\mathrm{C}$ Janelidze.

Kazbegi series

TB-46. Kazbegi, Georgian SSR

$6580 \pm 70$

$4630 \mathrm{BC}$

Peat, $4 \mathrm{~m}$ deep in diluvial terrace surface, from same location as TB-44, 45. Subm by C Janelidze.

TB-44. Kazbegi, Georgian SSR

$5950 \pm 90$ $4000 \mathrm{BC}$

Wood, 3.2m deep below terrace surface, from deposits of lst terrace over flood plain of Terek R, Goristsikhe village, Kazbegi region. Subm by C Janelidze, Inst Geog, Acad Sci, GSSR.

TB-45. Kazbegi, Georgian SSR

$\mathbf{3 4 7 0} \pm \mathbf{5 0}$ 1520 BC

Peat, $2 \mathrm{~m}$ deep, from same location as TB-44, from diluvial deposits from surface of peaty horizon. Subm by $\mathrm{C}$ Janelidze.

Sakorkio series

TB-55. Sakorkio, Georgian SSR

$6660 \pm 100$

Peat, $19 \mathrm{~m}$ deep, from Holocene deposits on left bank of Rioni R, Sakorkio village, Khobi region. Subm by C Janelidze.

TB-56. Sakorkio, Georgian SSR

$3150 \pm 90$

Peat, $6 \mathrm{~m}$ deep, from same location as TB-55. Subm by C Janelidze.

TB-57. Sakorkio, Georgian SSR

$31,300 \pm 320$ Janelidze. 
TB-65. Samikao, Georgian SSR

Peat, $67 \mathrm{~m}$ deep, from upper Pleistocene deposits, left bank of Tsivi $\mathrm{R}$, Samikao village, Abasha region. Subm by C Janelidze.

\title{
TB-66. Ureki, Georgian SSR
}

$35,300 \pm 690$

33,350 BC

Wood, $4 \mathrm{~m}$ deep in terrace surface, from sea deposits of late caragate terrace of Black Sea, Ureki village, Makharadze region. Subm by C Janelidze.

\section{TB-67. Anaklia, Georgian SSR}

$4050 \pm 50$

Peat, $3.5 \mathrm{~m}$ deep, from Anaklian peaty deposits, left bank of Tikori R, Anaklia village, Zugdidi region. Subm by C Janelidze.

\section{TB-69. Khorga, Georgian SSR}

$5600 \pm 50$

$3650 \mathrm{BC}$

Peat, $6 \mathrm{~m}$ deep, from lower layer of peaty horizon, mouth of Khobi R, Khorga village, Khobi region. Subm by C Janelidze.

\section{TB-70. Khorga, Georgian SSR}

$4170 \pm 50$ 2220 BC

Peat, $3.5 \mathrm{~m}$ deep, from upper layer of peaty horizon, from same location as TB-69. Subm by C Janelidze.

\section{TB-71. Kariata, Georgian SSR}

$910 \pm 40$

Wood, $8 \mathrm{~m}$ deep, Kariata village, Khobi region, mouth of Khobi R. Subm by C Janelidze.

\section{TB-76. Chakva, Georgian SSR}

$870 \pm 40$

Wood, $1.2 \mathrm{~m}$ deep in terrace surface, Chakva village, Kobuleti region, Ajarian SSR. Subm by C Janelidze.

\section{TB-78. Akhalkalaki, Georgian SSR}

$4865 \pm 60$

Peat, $3.75 \mathrm{~m}$ deep, Kartsakhi swamp, Kartsakhi village, Akhalkalaki region. Subm by $\mathrm{C}$ Janelidze.

TB-79. Akhalkalaki, Georgian SSR

Peat, 2.25m deep, from same location as TB-78. Subm by C Janelidze.

\author{
REFERENCES
}

Barker, Harold, Burleigh, Richard, and Meeks, Nigel, 1969, British Museum natural radiocarbon measurements VI: Radiocarbon, v 11, p 278-294.

1969, New method for the combustion of samples for radiocarbon dating: Nature, v 221, no. 5175, p 49-50.

Burchuladze, A A, 1968, Tbilisi radiocarbon dates I: Radiocarbon, v 10, p 466-467. Burchuladze, A A et al, 1969, Tbilisi radiocarbon dates II: Radiocarbon, v 11, p 499-501.

1970, New system for the combustion of samples for radiocarbon dating: The works of the Symposium "Astrophysical phenomena Radiocarbon", Tbilisi, p $51-55$ (in Russian).

1974, On some method of synthesis of the scintillation solvent from natural patterns containing carbon: The works of the Symposium "Astrophysical phenomena Radiocarbon", Tbilisi, p 317-323 (in Russian). 